Published in final edited form as:

J Psychopathol Behav Assess. 2018 September ; 40(3): 402-411. doi:10.1007/s10862-018-9666-6.

\title{
Reward Responsivity in Parenting: Development of a Novel Measure in Mothers of Young Children
}

\author{
Chelsey M. Hartley ${ }^{1}$, Jeremy W. Pettit ${ }^{2}$, Daniel M. Bagner², José R. Rosa-Olivares ${ }^{3}$ \\ Jeremy W. Pettit: jpettit@fiu.edu; Daniel M. Bagner: dbagner@fiu.edu; José R. Rosa-Olivares: Jose.Rosa- \\ Olivares@mch.com \\ ${ }^{1}$ Department of Psychology, Florida International University, Miami, FL, USA \\ ${ }^{2}$ Department of Psychiatry, University of Rochester Medical Center, Rochester, NY, USA \\ ${ }^{3}$ Nicklaus Children's Hospital, Miami, FL, USA
}

\section{Abstract}

The purpose of the current study was to develop and evaluate a measure of maternal reward responsivity in parenting. Deficits in reward responsivity, a common feature of depression, may contribute to maladaptive parenting behaviors. Reward responsivity is an individual difference in reactivity to pleasurable stimuli and represents a key motivational component that could contribute to the frequency and quality of mothers' interactions with their infants. However, there is currently no measure of reward responsivity in parenting, which would be necessary to evaluate the link between mother reward responsivity, behaviors towards their infant, and infant behavior. Therefore, the current study reports on the development and initial evaluation of a self-report measure of reward responsivity in parenting, the Mother Inventory of Reward Experience (MIRE). We evaluated the MIRE among 200 mothers $(M=28.45, S D=5.50)$ recruited from a pediatric primary care center. After item analysis, 22 items were retained and displayed high internal consistency reliability and test re-test reliability. Convergent validity was established via a significant correlation with global reward responsivity. Concurrent validity was established via significant correlations with depressive symptoms, parenting stress, and child behavior problems. Incremental validity of the MIRE over a measure of global reward responsivity was supported. These results support the reliability and validity of the MIRE as a measure of reward responsivity in parenting.

\footnotetext{
Chelsey M. Hartley chelsey_hartley@urmc.rochester.edu.

Compliance with Ethical Standards

Ethical Approval All procedures performed in studies involving human participants were in accordance with the ethical standards of the institutional and/or national research committee and with the 1964 Helsinki declaration and its later amendments or comparable ethical standards.

Informed Consent Informed consent was obtained from all individual participants included in the study.

Experiment Participants All participants provided written informed consent. Experimental protocols in this study were approved by the university and hospital's institutional review board and all procedures met the guidelines of the institutional and/or hospital research committees.

Conflict of Interest Chelsey Hartley, Jeremy Pettit, Daniel Bagner and José Rosa-Olivares all declare that they have no conflict of interest.
} 


\section{Keywords}

Reward responsivity; Parenting; Depressive symptoms

Research and theory highlight parenting behaviors as a potential mechanism of risk transmission for negative outcomes in offspring, including offspring of mothers with elevated depressive symptoms (Goodman and Gotlib 1999; Goodman et al. 2011). However, at present it is unclear what variables interfere with adaptive parenting behaviors. We propose low reward responsivity in parenting as a potential contributing variable to suboptimal parenting behaviors, especially in mothers with elevated depressive symptoms. Reward responsivity is an individual difference in reactivity to pleasurable stimuli and represents a key motivational component that could contribute to the frequency and quality of mothers' interactions with their infants. Because no measure of reward responsivity in parenting exists, the purpose of the present study was to develop the Mother Inventory of Reward Experiences (MIRE), a self-report measure of reward responsivity in parenting behaviors among mothers, and establish its reliability and validity.

Given evidence that parenting behaviors during mother- offspring interactions predict offspring negative outcomes (Cohn et al. 1990; Field et al., 1988; Goodman et al. 2011), intervening to improve parenting behaviors represents a potentially promising route to reduce the risk of negative offspring outcomes. Didactic instruction or direct modeling of adaptive parenting behaviors represents one intervention approach (Fewell and Wheeden 1998), but that approach assumes (a) a knowledge or skills deficit underlies maladaptive parenting behaviors and (b) the provision of knowledge or skills training will translate to positive parenting behaviors. Those remain unaddressed empirical assumptions. In the following section, we discuss reward responsivity as a potential contributing variable to suboptimal parenting behaviors.

\section{Reward Responsivity: a Potential Contributor to Suboptimal Parenting Behaviors}

Low positive affectivity (i.e., reward sensitivity) is the endophenotype most consistently associated with depression (Hasler et al. 2004). Positive affectivity is generated when an individual has a rewarding experience with the environment (Davidson 2000). Individuals find interpersonal rewards as the most salient and anticipated of all rewards and interpersonal rewards contribute to positive affectivity (Depue and Collins 1999; Depue and Morrone-Strupinsky 2005). Reward responsivity is a fundamental aspect of positive affectivity. It is defined as an individual difference in reactivity to pleasurable stimuli and reward (Bogdan and Pizzagalli 2009). Reward responsivity can be divided into an anticipatory phase (future wanting) and a consummatory phase (present liking; Henriques and Davidson 2000; Pizzagalli et al. 2008; Sherdell et al. 2012). Depressed individuals relative to nondepressed individuals show significantly lower levels of anticipatory pleasure in reward wanting and motivation to obtain reward (Berridge and Robinson 1998; Berridge and Robinson 2003; Sherdell et al. 2012). 
To our knowledge, the associations between reward responsivity, maternal depressive symptoms, and parenting behaviors have not been directly examined; however, higher levels of maternal depressive symptoms are associated with difficulties in motivation to engage with offspring and low levels of positive emotions during interactions with offspring, which are consistent with the possibility of deficits in maternal reward responsivity (Field et al. 2007; Lovejoy et al. 2000). Deficits in reward responsivity, to the extent they are present, may influence the behaviors displayed by mothers with elevated depressive symptoms during interactions with their infants, including low attentiveness to their infants, inconsistent responsiveness to their infants' signals of distress, and lower rates of smiling at and touching their infants (Forman et al. 2007; O'Hara 2009). For example, a mother with elevated depressive symptoms may not enjoy feeding their infant or interacting with their infant while changing diapers (e.g., singing a song to their infant) as much as a mother without depressive symptoms.

Identifying the presence of reward responsivity deficits and how they are associated with maternal depressive symptoms could inform models of risk transmission and potentially identify a target for interventions to improve parenting behaviors. Indeed, it is possible that didactic and/or modeling programs to improve parenting behaviors may not translate to the enactment of positive parenting behaviors if mothers experience deficits in anticipatory and consummatory pleasure related to interacting with their infants.

\section{The Need for a Measure of Maternal Reward Responsivity}

Efforts to identify reward responsivity deficits and examine how they may be associated with mother-infant interactions and maternal depressive symptoms are hindered by the lack of a measure of maternal reward responsivity in mother-child interactions. Prior to using resource intensive neuroscience methods to measure reward responsivity, such as fMRI paradigms (Forbes and Dahl 2012), it is important first to gather behavioral data to establish the presence of reward responsivity deficits with respect to parenting behaviors among mothers. We are aware of two self-report measures of global reward responsivity. The Snaith Hamilton Pleasure Scale (SHAPS, Snaith et al. 1995) is a 14-item scale that measures the capacity to experience pleasure in the past few days. The Tripartite Pleasure Inventory (TPI, Leventhal 2012) is a 36-item scale that measures hedonic responsivity, hedonic engagement, and hedonic desire on 12 common pleasant experiences.

Although measures of global reward responsivity exist, there is no measure that assesses reward responsivity specifically in the context of mother-infant interactions or parenting behaviors in general. The absence of a measure of reward responsivity specific to parenting behaviors represents a gap in the field that prevents advancement of research and theory on explanations of the associations between maternal depressive symptoms, mother-infant interactions, and child outcomes. The purpose of the present study was to address that gap by developing the Mother Inventory of Reward Experiences (MIRE), a self-report measure of reward responsivity in parenting behaviors among mothers, and establishing its reliability, factorial validity, convergent validity, concurrent validity, and incremental validity. Evidence in support of the reliability and validity of the MIRE will place the field in a better position 
to evaluate the links between mother reward responsivity and maternal behavior and test models of risk transmission in future studies.

\section{Method}

\section{Participants and Procedures}

For developmental piloting of MIRE items, we identified and recruited 12 English-speaking mothers with at least one child between the ages of 0 and 3 years. Children within this age range were recruited because the field of infant mental health refers to infancy as birth to 3 years (Zeanah 2009) and because transmission of risk is most likely to be identified early on via parenting behaviors with children in this age range. For full examination of the MIRE, we approached 373 mothers with at least one child between the ages of 0 and 3 years from a large outpatient pediatric primary care clinic housed in a pediatric hospital in an urban area in the Southeastern US. Of the 373 mothers approached to participate, 200 mothers (53.6\%) agreed to participate and provided informed consent, 73 mothers (19.6\%) declined to participate, and 100 mothers (26.8\%) were ineligible because they could not speak and read English fluently (they all were Spanish speaking). Participants ranged in age from 18 to 45 years $(M=28.45$ years; $S D=5.50$ years). The majority $(71.5 \%)$ were Hispanic and White (86.7\%); 9.7\% were African American; the remaining 3.6\% were Asian, Native American, or multi-racial. Approximately three-fourths of the sample was married or in a committed relationship (74.2\%), $22.7 \%$ were single, and $3.1 \%$ were separated or divorced. Education level was as follows: $8.2 \%$ did not complete high school, $19.1 \%$ completed high school, $31.4 \%$ completed some college or technical school, $25.3 \%$ completed college, and $16.0 \%$ earned a graduate degree. Mean annual income was $\$ 47,550(S D=\$ 37,457)$. Mean age of children was 14.82 months ( $S D=11.24$ months), ranging from 1 month to 36 months.

All 200 mothers were administered study measures in a quiet area of the waiting room of the pediatric primary care clinic. A subset of 20 mothers $(10 \%)$ were contacted by telephone two weeks later and re-administered the MIRE for the purpose of collecting data on testretest reliability. This study was approved by the Institutional Review Boards at both the pediatric hospital and affiliated university.

\section{Measures}

A Demographic and Background Questionnaire was administered to record data on age, race, ethnicity, marital status, employment status, household income, living situation, and current and prior mental health treatment.

\section{MIRE Item Pool Development}

Guided by the theory of reward responsivity, an initial item pool of 33 items was developed for each of two subscales corresponding to the phases of reward responsivity: anticipatory and consummatory, which we will refer to as Desire and Pleasure throughout. This resulted in a total of 66 items. The item stems were the same across both subscales, but participants were given different instructions and response options for each subscale. For the desire subscale, participants were given instructions to indicate: "how strongly you usually want to do the following experiences." The item response options were no desire, mild desire, 
moderate desire, great desire, and extreme desire. For the pleasure subscale, participants were given instructions to indicate: "how much pleasure, happiness, or enjoyment you usually feel in the moment while doing that type of experience." The item response options were no pleasure, mild pleasure, moderate pleasure, great pleasure, and extreme pleasure. When creating the item pool, we intentionally developed a range of items that parents would experience as more or less rewarding to ensure sufficient variability in responding (cf. Clark and Watson 1995); therefore, we included activities that may come across as unpleasant (e.g., "Cleaning up your child's mess"). Although this may not seem enjoyable, a mother can find cleaning up a child's mess as rewarding because the task is completed for their infant, even though they did not find the task itself enjoyable. By including items that were outside typical rewarding interactions, the item pool was diverse and allowed for variability in responding.

After the item pool was developed, doctoral-level psychologists with expertise in the areas of reward responsivity and depression, parenting, and psychometrics reviewed the item pool for appropriateness, representativeness, and explicitness. Based on their review, one subscale item was dropped, two subscale items were reworded and nine subscale items were added. Given that the item stems were the same across both subscales, when an item was removed from one subscale, it was also removed from the other subscale. The same was true when an item was added. These deletions and additions resulted in 82 items (41 for each subscale) for pilot testing. Twelve mothers were identified and invited to complete the self-report measure created from the modified item pool and to provide feedback on item length, wording, response choices, and content. From their feedback, 12 items were removed because they were difficult to understand or had content deemed irrelevant to the mothers' experiences. After the initial pilot testing was completed, 70 total items (35 items per subscale) were retained and were examined in the larger sample of 200 mothers.

\section{Measure for Evaluating Convergent Validity}

The Tripartite Pleasure Inventory (TPI, Leventhal 2012) is a self-report measure of global reward responsivity. The TPI consists of three subscales: hedonic responsivity, hedonic engagement and hedonic desire. For hedonic responsivity, respondents rate how much pleasure/happiness/enjoyment they usually feel for 12 pleasant experiences. For hedonic engagement, participants are asked to rate how often they usually engage in these 12 experiences. For hedonic desire, participants are asked to rate how strongly they usually want to engage in these 12 experiences. Cronbach's alpha in the current sample for hedonic responsivity, hedonic engagement, and hedonic desire was $a=.89, .89$, and .92 , respectively.

\section{Measures for Evaluating Concurrent Validity}

a. Center for Epidemiologic Studies Depression Scale (CESD, Radloff 1977) is a 20-item measure used to assess depressive symptom severity. Cronbach's alpha in the present sample was .86.

b. The Parent Stress Index, Short Form (PSI-SF, Abidin 1995)isa36-item measure that was used to measure total parenting stress. Cronbach's alpha for the PSI-SF total scale in the present sample was .93 . 
c1. For children under 12 months, the Infant Behavior Questionnaire-Revised Very Short Form (IBQ-R Very Short Form; Putnam et al. 2014) was administered to mothers as a measure of infant behavior problems. The IBQ-R Very Short Form is a 37-item parent-report questionnaire of infant temperament in specific situations during the past 1 to 2 weeks. It contains three subscales: Positive Affectivity/Surgency (PAS), Negative Emotionality (NEG), and Orienting/ Regulatory Capacity (ORC). Cronbach's alpha in the current sample for the PAS, NEG, and ORC subscales was a $=.69, .88$, and .66 , respectively.

c2. For children older than 12 months, the internalizing and externalizing domains of the Infant-Toddler Social and Emotional Assessment (ITSEA; Carter and Briggs-Gowan 2006) was administered to mothers as a measure of child behavior problems. In the current study, Cronbach's alpha for the externalizing domain was .91, and Cronbach's alpha for the internalizing domain was .87.

\section{Data Analysis}

Missing data was present on $13.26 \%$ of variables. To assess missing data bias, a dummy variable reflecting the present or absence of missing data for each variable was created and correlated with all other study variables. No correlation was statistically significant, indicating no evidence of bias. Data were assumed to be missing at random. Multiple imputation averaging across 10 imputation sets was used to accommodate missing data (Little and Rubin 1989).

Item Analysis-The purpose of item analysis was to find the items that formed an internally consistent scale and to eliminate the items with poor measurement ability (Spector 1992). MIRE item scores ranged from 0 to 4 . We examined the mean and kurtosis of each item. Consistent with recommendations on measurement development (Allen and Yen 1979; Spector 1992), items with extreme means (below .25 or above 3.75 ) and high kurtosis (values $>|3|$ ) were deleted.

Item-Total Correlations-We examined item-total correlations to evaluate how well each item related to other items in the same subscale. Item-total correlations were examined by calculating the item-remainder coefficient for each item. The item remainder coefficient is the correlation of each item with the sum of the remaining items in the subscale. Following recommendations for measurement development (Allen and Yen 1979; Spector 1992), items with item-remainder coefficients less than .30 were removed to create a scale with highinternal consistency.

Exploratory Factor Analysis-An exploratory factor analysis (EFA) was performed using principal component analysis with an oblique rotation to identify the factor structure and whether the identified factors were consistent with the intended subdimensions in the MIRE. Oblique rotation was used because we expected that factors would be correlated. Parallel analysis was performed by means of syntax created and made available by O'Connor (2000). One thousand randomized permutations were used to create a set of the mean eigenvalues and the corresponding 95th percentile eigenvalues. The eigenvalues from the raw data were retained if they were greater than the 95 th percentile eigenvalues created 
from the randomized dataset. Once the number of factors was determined, a coefficient level of .32 or above was used to determine if an item loaded on a given factor (Costello and Osborne 2005). In this initial examination of the MIRE, we did not remove items based on factor loadings, as it is recommended to use a normative data sample to delete items using an EFA (Spector 1992).

Reliability-We created a final measure after removing all items with poor psychometric properties based on recommendations described above. Using the final measure, internal consistency reliability was estimated using Cronbach's alpha and testretest reliability over two weeks was estimated using Intraclass Correlation Coefficients (ICC).

Validity-Convergent validity was examined by calculating Pearson product moment correlations between scores on the MIRE and a measure of global reward responsivity, the TPI. Concurrent validity was examined by calculating Pearson product moment correlations between scores on the MIRE and the CES-D, the PSI, the IBQ PAS, NEG and ORC subscales, as well as the ITSEA INT and EXT subscales. Incremental validity was examined by conducting regression models in which the MIRE predicted parenting stress measured by the PSI and child outcomes measured by the IBQ or IITSEA, while covarying TPI scores.

\section{Results}

\section{Item Analysis and Item-Total Correlations}

The range of scores on each item of the MIRE was 0 to 4 . Across the subscales, 46 items (23 items from eachsubscale) were deleted for extreme means or high kurtosis values. Example deleted items include: "Playing with your child," "Tickling your child," "Singing to your child." Four items ( 2 items from each subscale) were deleted because they had itemremainder coefficients less than .30. These items were "Listening to your child cry" and "Punishing your child." The remaining 22 items (11 on each subscale) were retained for examinations of factor structure, reliability, and validity.

\section{Exploratory Factor Analysis (EFA)}

An EFA on the final 22 items was conducted using principal components analysis. The eigenvalues from the raw data were retained as a factor if they were greater than the 95th percentile eigenvalues created from the randomized data (O'Connor 2000). Figure 1 presents the scree plot of the raw data, mean, and 95th percentile eigenvalues. There were four eigenvalues computed from the original raw data greater than the 95th percentile eigenvalues data. According to parallel analysis, any factors that are above the intersection in the actual data and the 95th percentile line should be retained (O’Connor 2000). In Fig. 1, four factors are above the intersection. Table 1 presents the factor loadings for each of the four factors, with the highest factor loading bolded for each item. Although four factors emerged, all items had loadings > .32 on the first factor and all but three items had highest loading on the first factor. The percentages of variance accounted for by the four factors were $44.73 \%$, $9.24 \%, 7.39 \%$, and $5.04 \%$, respectively. The pattern of factor loadings and the percent of variance accounted for by the first factor suggest a single factor may best explain the variance in maternal reward responsivity on the MIRE. 


\section{Reliability}

Internal consistency reliability was examined using Cronbach's alpha for the 22-item total measure, and was found to be .94. Cronbach's alpha for the Desire and Pleasure 11-item subscales was .87 and .86 , respectively. Test-retest reliability over two weeks for the MIRE total score was ICC $=.93(p<.01)$, the MIRE Desire scores was ICC $=.95(p<.01)$, and the MIRE Pleasure scores was ICC $=.92(p<.01)$.

Validity

Table 2 shows the means of, standard deviations of, and the intercorrelations between the MIRE total score, MIRE Desire and Pleasure subscales, and all other measured variables. In support of convergent validity, the MIRE total scale was significantly and positively correlated with the TPI Desire subscale, $r=.26, p<.001$ and the TPI Pleasure subscale, $r=$. $33, p<.001$. The MIRE Desire subscale was significantly and positively correlated with TPI Desire subscale, $r=.29, p<.001$, and the MIRE Pleasure subscale was significantly and positively correlated with TPI Pleasure subscale, $r=.27, p<.001$. In support of concurrent validity, the MIRE total score, $r=-.20, p<.01$, the MIRE Desire subscale, $r=-.17, p=.02$, and the MIRE Pleasure subscale, $r=-.20, p=.01$, were significantly and negatively correlated with maternal depressive symptoms measured using the CES-D. In further support of concurrent validity, the MIRE total score, $r=-.39, p<.001$, the MIRE Desire subscale, $r=-.36, p<.01$, and the MIRE Pleasure subscale, $r=-.28, p<.01$, were significantly and negatively correlated with parenting stress measured using the PSI.

Finally, in partial support of concurrent validity, the MIRE total score and the MIRE Desire and Pleasure subscales were significantly correlated with some, but not all, measures of child behavior problems. Among 100 mothers of children younger than 12 months of age, the MIRE total score and the MIRE Desire subscale were significantly correlated with the IBQ Positive Affectivity/Surgency $(r=.27, p=.03 ; r=.30, p=.03)$ and IBQ Orienting/ Regulatory Capacity $(r=.43 p<.001 ; r=.38 p=.01)$, but not the IBQ Negative Emotionality $(\mathrm{r}=-.18, p=.16 ; \mathrm{r}=-.23, p=.09)$. The MIRE Pleasure subscale was not significantly correlated with the IBQ Positive Affectivity/Surgency $(r=.18, p=.22)$ or IBQ Negative Emotionality ( $\mathrm{r}=-.12, p=.40)$, but was significantly correlated with the IBQ Orienting/ Regulatory Capacity $(r=.37, p=.01)$. Among 100 mothers of children older than 12 months of age, the MIRE total scale were significantly and negatively correlated with the ITSEA Externalizing $(r=-.24, p=.03)$ and ITSEA Internalizing $(r=-.37, p=.001)$. The MIRE Desire subscale and the MIRE Pleasure subscale were significantly correlated with the ITSEA Externalizing $(r=-.37, p=.06 ; r=-.26, p=.03)$, but not the ITSEA Internalizing $(r$ $=-.21, p=.09 ; r=-.20, p=.10)$.

To examine the incremental validity of the MIRE, six separate hierarchical regression analyses were constructed using the MIRE total score as the predictor variable, while covarying TPI scores, child age, and child sex. The six hierarchical regression analyses used the following outcome variables as the dependent variable, respectively: PSI Total Stress score, the three IBQ subscales (PAS, NEG, ORC), and the two ITSEA subscales (EXT, INT). The MIRE significantly incrementally predicted parenting stress (Table 3 ) and infant behavior problems measured using the IBQ Positive Affectivity/Surgency (PAS) subscale 
(Table 3) and the IBQ Orienting/Regulatory Capacity (ORC) subscale (Table 3). The MIRE did not significantly incrementally predict the IBQ NEG $(\mathrm{B}=-.35 \pm .34, p=.30)$, the ITSEA Intemalizing ( $\mathrm{B}=-.05 \pm .06, p=.34)$, or the ITSEA Externalizing $(\mathrm{B}=-.11 \pm .06, p=.08$ ).

\section{Discussion}

Prior to the current study, no measure of reward responsivity in parenting existed. It was important to develop such a measure to help future research unpack the link between reward responsivity and parenting behavior among mothers with depression. Maternal depression is associated with difficulties in parenting behavior, including: motivation to engage with offspring and low levels of enjoyment during interactions with offspring. These are consistent with the possibility of deficits in maternal reward responsivity. Therefore, developing and testing the MIRE was an important first step to empirically evaluate the link between reward responsivity, parenting behavior, and maternal depressive symptoms in future research.

The findings from the current study provide preliminary support for the reliability and validity of a new measure of reward responsivity in parenting, the MIRE. The MIRE is based on a theory of reward responsivity, applied to the context of parenting behaviors. In a sample of 200 mothers of young children, the MIRE total score and its two subscales, Desire and Pleasure, demonstrated high internal consistency reliability and high retest reliability over a two-week period. Further, convergent validity was supported via a significant association with a measure of global reward responsivity and concurrent validity was largely supported via significant associations with maternal depressive symptoms, parenting stress, and measures of infant and child behavior problems. Finally, incremental validity was partially supported via significant incremental predictive associations with parenting stress and infant behavior problems, while controlling for global reward responsivity. These findings thus provide initial support for the use of the MIRE as a measure of reward responsivity in parenting behaviors.

With the MIRE, researchers will be able to measure and empirically evaluate the potential associations between maternal reward responsivity and maternal behavior to inform models of risk transmission and the development and evaluation of interventions targeting parenting behaviors among mothers with elevated depressive symptoms. As noted, deficits in reward responsivity may influence the behaviors displayed by mothers during interactions with their children, including low attentiveness to their children, inconsistent responsiveness to their children's signals of distress, and lower rates of smiling at and touching their young children (Forman et al. 2007; O'Hara 2009). For example, during a feeding interaction, a nondepressed mother may find the interaction very rewarding and want to interact with her infant whereas a mother with depression may find the interaction taxing. If that is the case, then developing interventions to enhance reward responsivity would represent a potentially promising route to reduce risk transmission and enhance positive outcomes in young children of mothers with elevated depressive symptoms. For example, regulating responses to rewards is a potential pathway for intervention. 
The majority of research on reward responsivity has used performance-based reward tasks. The MIRE offers a practical, easy to administer, and inexpensive complement to those tasks. The MIRE can be used to identify maternal reward responsivity deficits and examine their associations with relevant outcomes such as parenting behaviors or child behavior problems. As research on the MIRE progresses, it will be important to examine the correspondence between scores on the MIRE and performance based-reward tasks.

Based on theory and past research, the MIRE was developed with subscales representing an anticipatory phase (Desire) and a consummatory phase (Pleasure; Henriques and Davidson 2000; Pizzagalli et al. 2008; Sherdell et al. 2012). Findings from exploratory factor analyses on the MIRE did not align with theory driven structure of the MIRE, but instead suggested a single factor may most parsimoniously explain variance in reward responsivity in parenting as measured using the MIRE. One possible explanation of these findings is that reward responsivity in parenting is a unitary construct (i.e., anticipatory and consummatory phases are not separate factors), which would necessitate a revision to the theoretical model. An alternative explanation is that mothers may have difficulty reflecting on their internal experiences and feelings in a way that allows them to differentiate anticipatory and consummatory phases of reward. Additional research with a larger normative sample will be needed to further examine the MIRE subscales and to draw conclusions on the structure of reward responsivity in parenting. Given the absence of support for the two-factor structure of the MIRE in this study, total scores on the MIRE should be given preference over the subscale scales.

Future research will need to evaluate the utility of the MIRE in clinical settings. A tool for assessing maternal reward responsivity could help clinicians evaluate the degree to which a mother experiences desire and pleasure from interactions with her infant. With the MIRE, a clinician could evaluate the efficacy of interventions aimed at increasing rewarding experiences during parent-child interactions. The MIRE would enable clinicians to examine the changes in maternal reward responsivity pre and post treatment.

This study's findings should be interpreted in light of its limitations. First, the measurement approach relied solely on rating scales completed by a single informant (mothers). Given the purpose of the current study was measurement development and evaluation, we did not directly measure parenting behavior or evaluate a conceptual model wherein reward responsivity accounts for the association between depression on parenting. Future studies should consider using behavioral observation tasks to examine the concurrent validity of the MIRE. For example, the Dyadic Parent-Child Interaction Coding System (DPICS; Eyberg et al. 2005)couldbe used to behaviorally code the quality of parent-child dyadic interactions. Associations between reward responsivity in parenting and positive maternal behaviors coded from the DPICS could be used to examine concurrent validity and the potential mediating effect of reward responsivity in mothers between depression and parenting behaviors. Future studies should also consider using a performance-based reward task to measure reward responsivity (e.g., Pizzagalli et al. 2005)and further examine the convergent validity of the MIRE. Finally, the present sample comprised of predominantly Hispanic/ Latina mothers. The generalizability of findings to other populations is unknown. 
Despite these limitations, this present study provides the first empirical data on a novel measure of reward responsivity in parenting. These findings support the use of the MIRE as a self-rating scale of reward responsivity in parenting among mothers. The brevity of the scale makes it easy to administer across a variety of settings, such as pediatric primary care. Future research is encouraged to further examine the factor structure of the MIRE in larger and diverse samples and to examine maternal reward responsivity as a possible explanatory variable of the association between maternal depressive symptoms and parenting behaviors.

\section{Acknowledgements}

We thank Drs. Chockalingam Viswevaran, Andy Pham, and Adam Leventhal for their contributions to this manuscript.

Funding This study was not funded by a grant.

\section{References}

Abidin RR (1995). Parenting stress index manual, third edition: Professional manual. Odessa: Psychological Assessment Resources, Inc.

Allen MJ, \& Yen WM (1979). Introduction to measurement theory. Monterey: Brooks/Cole Pub. Co..

Berridge KC, \& Robinson TE (1998). What is the role of dopamine in reward: Hedonic impact, reward learning, or incentive salience?

Brain Research Reviews, 28(3), 309-369. 10.1016/S0165-0173(98)00019-8.

Berridge KC, \& Robinson TE (2003). Parsing reward. Trends in Neurosciences, 26(9), 507-513. 10.1016/S0166-2236(03)00233-9. [PubMed: 12948663]

Bogdan R, \& Pizzagalli DA (2009). The heritability of hedonic capacity and perceived stress: A twin study evaluation of candidate depressive phenotypes. Psychological Medicine, 39(2), 211-218. 10.1017/S0033291708003619. [PubMed: 18507876]

Carter AS, \& Briggs-Gowan MJ (2006). ITSEA/BITSEA: Infant toddler and brief infant toddler social and emotional assessment examiner's manual. San Antonio: Harcourt Assessment.

Clark LA, \& Watson D (1995). Constructing validity: Basic issues in objective scale development. Psychological Assessment, 7(3), 309-319. 10.1037/1040-3590.7.3.309.

Cohn JF, Campbell SB, Matias R, \& Hopkins J (1990). Face-to-face interactions of postpartum depressed and nondepressed mother- infant pairs at 2 months. Developmental Psychology, 26(1), 15-23. 10.1037/0012-1649.26.1.15.

Costello AB, \& Osborne JW (2005). Best practices in exploratory factor analysis: Four recommendations for getting the most from your analysis. Practical Assessment, Research and Evaluation, 10(7), 1-9.

Davidson J (2000). Affective style, mood, and anxiety disorders : An affective neuroscience approach

Depue RA, \& Collins PF (1999). Neurobiology of the structure of personality: Dopamine, facilitation of incentive motivation, and extraversion. The Behavioral and Brain Sciences, 22(3), 491-517 discussion 518-469. [PubMed: 11301519]

Depue RA, \& Morrone-Strupinsky JV (2005). A neurobehavioral model of affiliative bonding: Implications for conceptualizing a human trait of affiliation. The Behavioral and Brain Sciences, 28(3),313-350; discussion 350-395 10.1017/s0140525×05000063. [PubMed: 16209725]

Eyberg SM, Nelson MM, Duke M, \& Boggs SR (2005). Manual for the dyadic parent-child interaction coding system (3rd ed.). Unpublished manuscript, Department of Clinical and Health Psychology, University of Florida, Gainesville, FL.

Fewell RR, \& Wheeden CA (1998). A pilot study of intervention with adolescent mothers and their children: A preliminary examination of child outcomes. Topics in Early Childhood Special Education, 18(1), 18-25. 10.1177/027112149801800105. 
Field T, Healy B, Goldstein S, Perry S, Bendell D, Schanberg S,... Kuhn C. (1988). Infants of depressed mothers show "depressed" behavior even with nondepressed adults. Child Development, 59(6), 1569-1579. 10.2307/1130671. [PubMed: 3208568]

Field T, Hernandez-Reif M, Diego M, Feijo L, Vera Y, Gil K, \& Sanders C (2007). Still-face and separation effects on depressed mother-infant interactions. Infant Mental Health Journal, 28(3), 314-323. 10.1002/imhj.20138. [PubMed: 28640469]

Forbes EE, \& Dahl RE (2012). Research review: Altered reward function in adolescent depression: What, when and how? Journal of Child Psychology and Psychiatry, 53(1), 3-15. 10.1111/j. 1469-7610.2011.02477.x. [PubMed: 22117893]

Forman DR, O'Hara MW, Stuart S, Gorman LL, Larsen KE, \& Coy KC (2007). Effective treatment for postpartum depression is not sufficient to improve the developing mother-child relationship. Development and Psychopathology, 19(2), 585-602. 10.1017/S0954579407070289. [PubMed: 17459185]

Goodman SH, \& Gotlib IH (1999). Risk for psychopathology in the children of depressed mothers: A developmental model for understanding mechanisms of transmission. Psychological Review, 106(3), 458-490. 10.1037/0033-295X.1063.458. [PubMed: 10467895]

Goodman SH, Rouse MH, Connell AM, Broth MR, Hall CM, \& Heyward D (2011). Maternal depression and child psychopathology: A meta-analytic review. Clinical Child and Family Psychology Review, 14(1), 1-27. 10.1007/s10567-010-0080-1. [PubMed: 21052833]

Hasler G, Drevets WC, Manji HK, \& Charney DS (2004). Discovering endophenotypes for major depression. Neuropsychopharmacology, 29(10), 1765-1781. 10.1038/sj.npp.1300506. [PubMed: 15213704]

Henriques JB, \& Davidson RJ (2000). Decreased responsiveness to reward in depression. Cognition and Emotion, 14(5), 711-724. 10.1080/02699930050117684.

Leventhal AM (2012). Relations between anhedonia and physical activity. American Journal of Health Behavior, 36(6), 860-872. 10.5993/AJHB.36.6.12. [PubMed: 23026043]

Little RJA, \& Rubin DB (1989). The analysis ofsocial-science data with missing values. Sociological Methods \& Research, 18(2-3), 292-326. 10.1177/0049124189018002004.

Lovejoy MC, Graczyk PA, O’Hare E, \& Neuman G (2000). Maternal depression and parenting behavior: A meta-analytic review. Clinical Psychology Review, 20(5), 561-592. 10.1016/ S0272-7358(98)00100-7. [PubMed: 10860167]

O'Connor BP (2000). SPSS and SAS programs for determining the number of components using parallel analysis and Velicer's MAP test. Behavior Research Methods, Instruments, \& Computers, 32(3), 396-402.

O'Hara MW (2009). Postpartum depression: What we know. Journal of Clinical Psychology, 65(12), 1258-1269. 10.1002/jclp.20644. [PubMed: 19827112]

Pizzagalli DA, Jahn AL, \& O'Shea JP (2005). Toward an objective characterization of an anhedonic phenotype: A signal-detection approach. Biological Psychiatry, 57(4), 319-327. 10.1016/ j.biopsych.2004.11.026. [PubMed: 15705346]

Pizzagalli DA, Iosifescu D, Hallett LA, Ratner KG, \& Fava M (2008). Reduced hedonic capacity in major depressive disorder: Evidence from a probabilistic reward task. Journal of Psychiatric Research, 43(1), 76-87. 10.1016/j.jpsychires.2008.03.001. [PubMed: 18433774]

Putnam SP, Helbig AL, Gartstein MA, Rothbart MK, \& Leerkes E (2014). Development and assessment of short and very short forms of the infant behavior questionnaire-revised. Journal of Personality Assessment, 96(4), 445-458. 10.1080/00223891.2013.841171. [PubMed: 24206185]

Radloff LS (1977). The CES-D scale: A self-report depression scale for research in the general population. Applied Psychological Measurement, 1(3), 385-401. 10.1177/014662167700100306.

Sherdell L, Waugh CE, \& Gotlib IH (2012). Anticipatory pleasure predicts motivation for reward in major depression. Journal of Abnormal Psychology, 121(1), 51-60. 10.1037/a0024945. [PubMed: 21842963]

Snaith RP, Hamilton M, Morley S, Humayan A, Hargreaves D, \& Trigwell P (1995). A scale for the assessment of the hedonic tone: The snaith-Hamilton pleasure scale. The British Journal of Psychiatry, 167(1), 99-103. 10.1192/bjp.167.1.99. [PubMed: 7551619] 
Spector PE (1992). Summated rating scale construction: An introduction. Thousand Oaks, CA, US: Sage Publications, Inc.

Zeanah CH (2009). Handbook ofinfant mental health (3rd ed.). New York, NY, US: Guilford Press. 


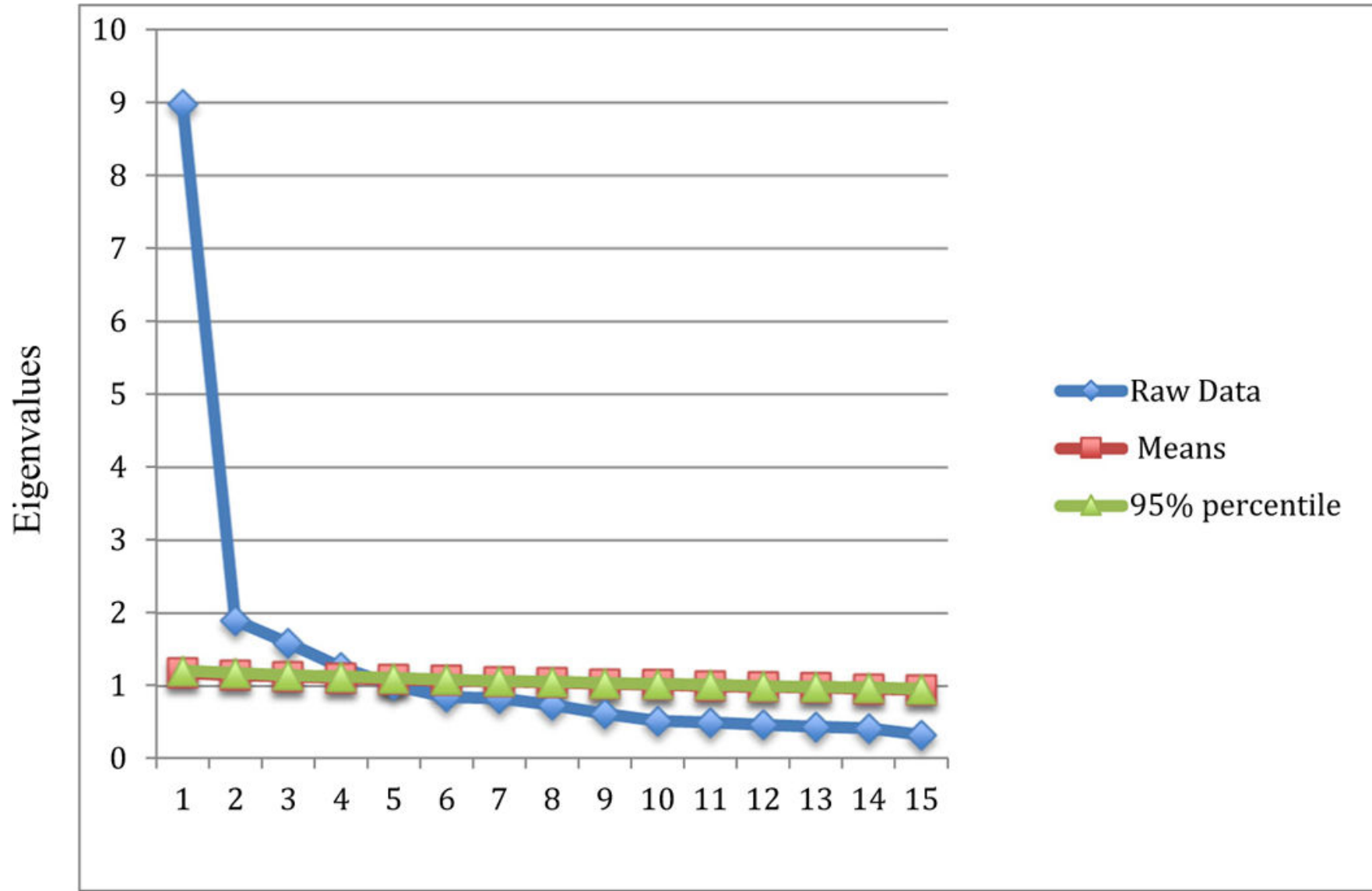

Factor \#

Fig.1.

Scree plot from the EFA with parallel analysis 


\section{을




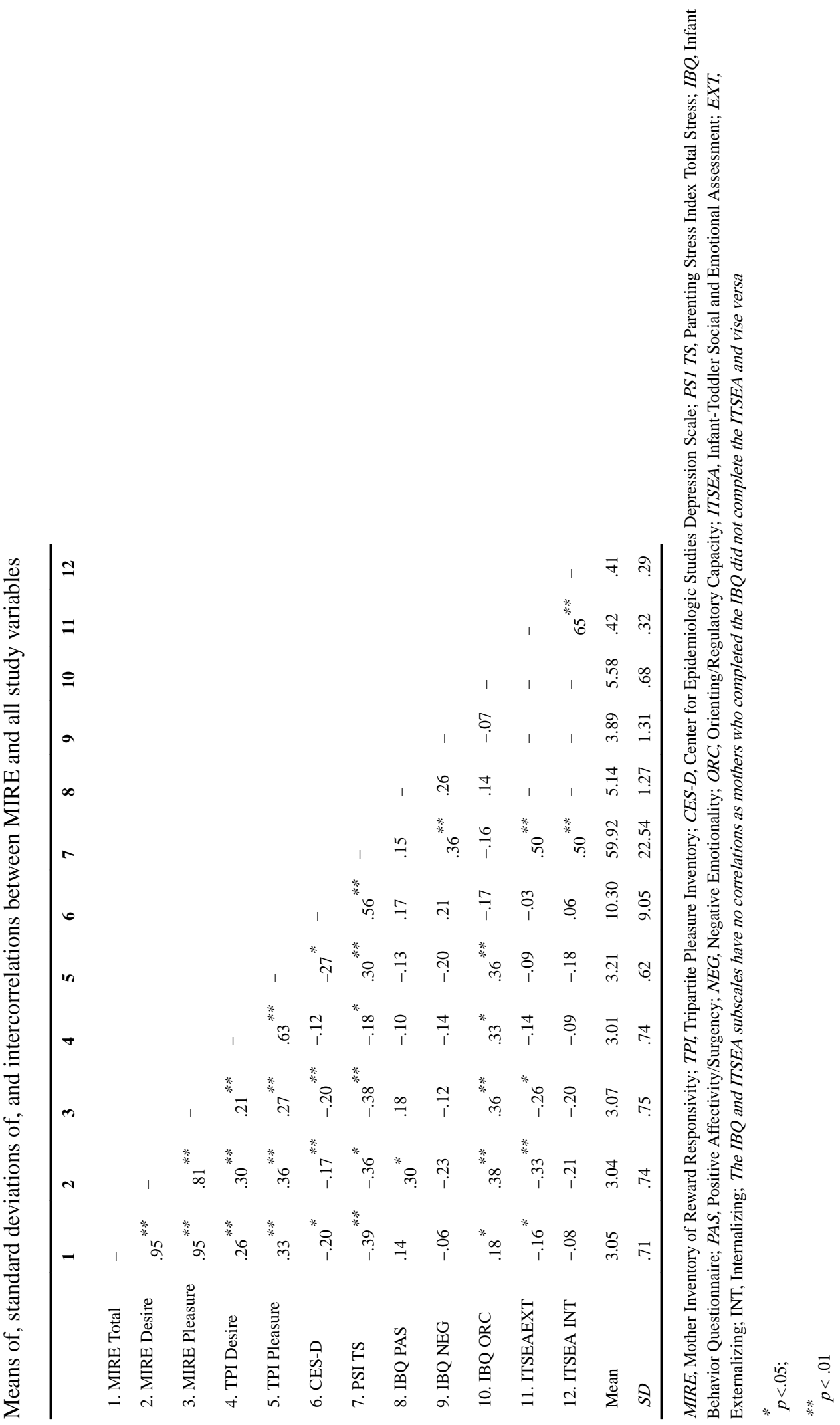




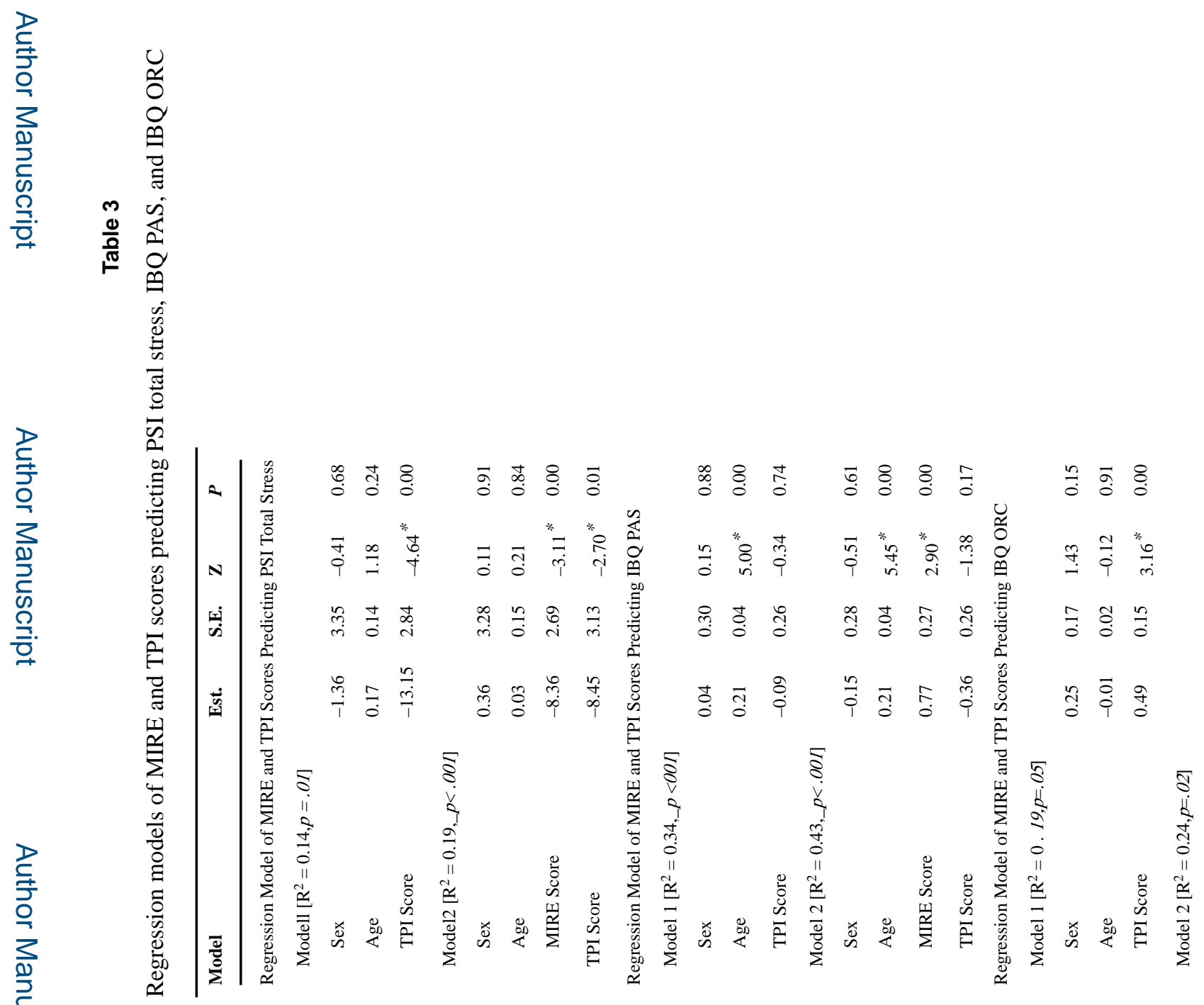




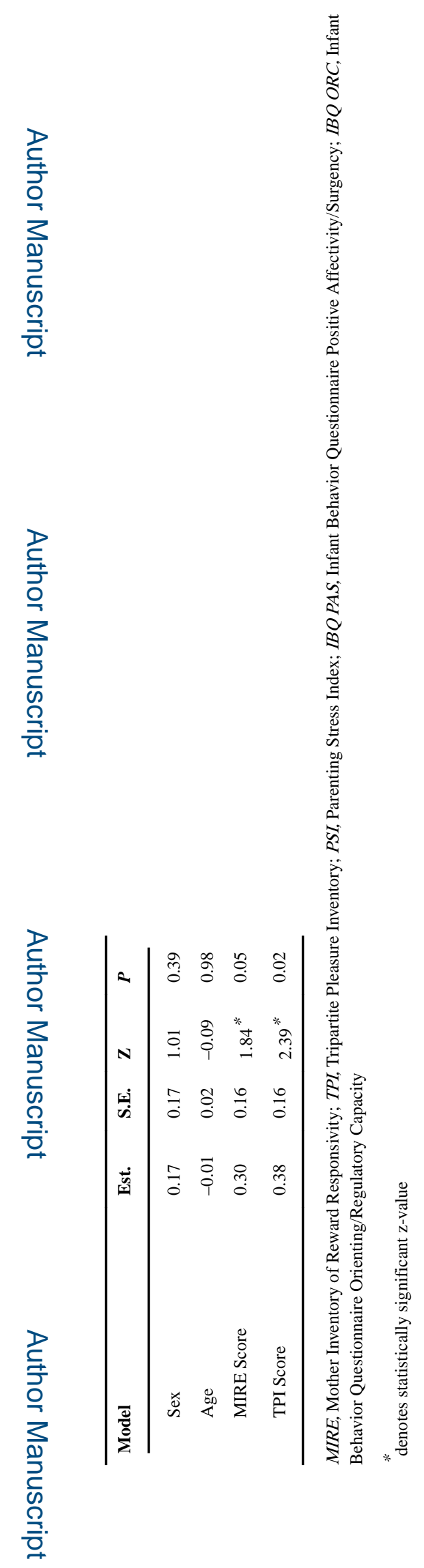

